\title{
Robust Interactive Storytelling Framework for Automatic TV Content/Story Production
}

\author{
Hussein Karam Hussein Abd El-Sattar \\ Ain Shams University, Faculty of Science, Mathematics and Computer Science Department, \\ Abbassia 11566, Cairo, Egypt \\ huss20@hotmail.com
}

\begin{abstract}
With the rapid advancement of hardware and software, entertainment computing industry has been popularized during the last decade. TV content/story production is a major bottleneck in the entertainment industry. Previous work has identified key problems, such as narrative control, the duality between character and plot, the tension between interactivity and storytelling, and the tools that artists can use to create interactive story worlds. Due to the tradeoff between narrative progression and user interaction, striking a balance between the two is a big challenge. As a solution to such a challenge, Bcreative system has been developed. It is a unified prototype of both plot-based and character-based interactive storytelling. The idea is derived from the study of both Petri nets (PN) and cinematography in interactive storytelling. The system architecture is composed of two engines: a story engine and a character engine through a smart user-friendly graphical user interface (GUI). The story engine models the story plot based on the Petri nets $(\mathrm{PN})$ reachability analysis, while the character engine enhances the modeling of character behavior based on filmmaking theory. This paper discusses the development process of our proposed system, illustrating the design and implementation in detail. Comparisons with existing techniques are included, and the advantages of our proposed system over existing ones are examined. Evaluation and practical performance results are also demonstrated.
\end{abstract}

\section{Introduction}

Interactive storytelling (IS) is a promising growth area in computer-based technologies, in the entertainment computer industry, and other applied areas. TV content/story production is a major bottleneck in the entertainment industry. The internet has certainly created a major stir, but the fact remains that TV content/story generation is still the primary means by which most people around the world get their information. It is generally assumed that individuals cannot easily produce TV content/story with conventional techniques because content production greatly consumes time, effort, budgets and it is not only limited to producers, directors, actors/actresses, etc., but also requires some level of explicit narrative representation and narrative control. The plot of the story and the character(s) are the two most important elements of interactive storytelling. In the field of interactive storytelling technology, we try to bridge a connection between player desires and story content to provide a deep connection between what the player does in the story world and where 
the story leads. Due to the tradeoff between story narrative and user interactions, striking a balance between them as well as the duality between character and plot throws up an aspiring goal and challenging task. As a solution to such a challenge, Bcreative system has been developed. It is a unified prototype of both plot-based and character-based interactive storytelling. The system structure is composed of two engines: a story engine and a character engine through a smart user-friendly graphical user interface. The story engine models the story plot based on the Petri nets (PN) reachability analysis $[5,14,18]$, while the character engine enhances the modeling of character behavior based on filmmaking theory $[4,9,6]$. The approach used a Petri nets as a state-based model for story plot generation, where each scene in a plot is regarded as a goal to be reached. A complex scene is represented as a composite goal, and a simple scene is represented as an atomic goal.

This paper is organized as follows. Section 2 introduces some terminology and basic notions that will be used. Section 3 outlines the previous relevant work on interactive storytelling technology and the problems that were encountered. The development process of our proposed system including system structure, methodology, design, etc. is discussed in section 4. System configuration is given in section 5 . Evaluation and practical performance results with comparisons are discussed in section 6. Section 7 concludes this paper and outlines some directions for future work.

\section{Background and Basic Notions}

For clarity and accuracy, this section summarizes the background and the basic terminology and notions that will be used throughout this paper.

\subsection{Interactive Storytelling Background}

Interactive storytelling is a developing kind of computer entertainment in which the player plays the role of the protagonist in a dramatically rich environment. In traditional forms of storytelling, a storyteller would present the scenario of a story to the audiences in a predefined way (also known as a plot), which limited the variation in character interactions and context. The story refers to the succession of actions that happen in the world represented by the narrative. A narrative means a certain type of artistic and social expression, where a kind of imitation of real events is involved. In dramatic writing, stories are thought of as consisting of events that turn (change) values. A value is a property of an individual or relationship, such as trust, hope, etc. Semantically, a scene (story event) is an atom of a story plot, which involves the interactions among a number of virtual actors with dedicated roles, under a certain context. An actor is the virtual entity which performs the tasks assigned from the drama manager (director). Generally, scenes are composed of beats, where the term beat refers to the smallest unit of action that has its own complete shape. A beat is a dramatic action that occurs in a scene to achieve a narrative goal. A beat consists of (1) preconditions, a list of predicates that need to be true for the beat to be selected; (2) post conditions; a list of predicates that will be true as a consequence of firing the beat; (3) success and failure conditions, and (4) a joint plan to be executed by the actors. Beats are the fundamental unit of actor guidance and it defines the granularity of plot/actor interaction. It serves several functions and plays an important role within the structure of our proposed interactive storytelling framework. 


\subsection{Cinematography and Interactive Drama Technology}

Drama is a special kind of narrative where the actions are directly represented to the spectator. There have been three main approaches in creating interactive drama technology: "Branching Narrative (Lots of story, little interactivity)", "Narrative Game (Lots of interactivity, little story)", and "Storytronics (Lots of both story and interactivity)". In Storytronics technology, interactive storytelling is achieved through artistic works called story worlds. A story world is a universe of dramatic possibilities. A story world is played by a player, who controls the protagonist actor in that story, with the other actors controlled by the computer. It is comprised of two groups of information: (i) dramatic components, and (ii) dramatic principles. Dramatic components are the same things that make up any story - the story's actors, locations, props, and occurrences. Dramatic principles are the unique way in which a story builder expresses his or her authorial voice. A story world is built up of many kinds of elements. These elements are divided into three broad categories: Deikto words, behavior controllers, and scripts. Behavior controllers are story world elements which are invisible to the player, but which affect the way story world actors behave. Deikto is a form of simplified English used by the storyteller. Deikto words are story world elements which the player can come in direct contact with. A Deikto sentence describes a dramatic action taken by an actor in the story world. If past, it is called an event, if planned for the future it is called a plan. Storyworlds contains all the concrete pieces that are used to build up the story such as storyworld's actors, places, stages, props as well as past and present occurrences and future plans. On the other hand, scripts are applied by actors at certain times, causing changes in the storyworld or directing actors on how to behave. Scripts are created using a special scripting language. This language is built out of operators, the same way that human language is built out of words. Bcreative uses a modified TVML as a cinematic and scripting language [6].

\subsection{Petri Nets (PN)}

A Petri nets (PN) $[14,18]$ is an abstract, formal model of information flow. It is a graphical and mathematical modeling tool applicable to many systems. As a graphical tool, PN can be used as a visual-communication aid similar to flow charts, block diagrams, and networks. As a mathematical tool, it is possible to set up state equations, algebraic equations, and other mathematical models governing the behavior of systems. A Petri nets $P N=\left(N, M_{0}\right)$ consists of a structure $\mathrm{N}$ and an initial marking $M_{0}$, where: $\mathrm{N}=(\mathrm{P}, \mathrm{T}, \mathrm{F}, \mathrm{W})$ is a Petri nets structure,

$P=\left\{p_{1}, p_{2}, \cdots, p_{m}\right\}$ is a finite set of $m$ places,

$T=\left\{t_{1}, t_{2}, \cdots, t_{n}\right\}$ is a finite set of $\mathrm{n}$ transitions,

$F \subseteq(P \times T) \cup(T \times P)$ is a set of arcs, where $P \cap T=\phi, P \cup T \neq \phi$.

$W: F \rightarrow\{1,2,3, \cdots\}$ is a mapping which associates to each arc of the net its weight, $M_{0}: P \rightarrow\{1,2,3, \cdots\}$ is the initial marking representing the initial state of PN. 
A special $(\mathrm{PN})$ in which place capacities and arc weights are equal to one is called a condition/event net (C/E net). A transition without any input place is called a source transition and one without any output place is called a sink. Labels for unitary weight are usually omitted. In modeling using PN, we regard the places as conditions and the transitions as events. On the other hand, the state of $\mathrm{PN}$ is described by means of the concepts of marking. A marking is a function that assigns to each place a nonnegative integer called a token. A token is a primitive concept of PN like places and transitions. From a graphic point of view, places are usually represented by circles, transitions by rectangles and marks by black dots into places. The dynamics of the net is described by moving tokens among places according to the following transition firing rules:

1. A transition $t$ is said to be enabled if each input place $p$ of $t$ is marked with at least $\mathrm{W}(\mathrm{p}, \mathrm{t})$ tokens, where $\mathrm{W}(\mathrm{p}, \mathrm{t})$ is the weight of the arc from $\mathrm{p}$ to $\mathrm{t}$.

2. An enabled transition may or may not fire depending on whether or not the event actually takes place.

3. A firing of an enabled transition $t$ removes $W(p, t)$ tokens from each input place $p$ of $t$ and adds $W(p, t)$ tokens to each output place of $t$, where $W(p, t)$ is the weight of the arc from $p$ to $t$.

4. The marking of other places which are neither inputs nor outputs of t remains unchanged.

\section{Problem Statement and Prior Research}

\subsection{Problem Statement}

Story plot and character(s) are the two most important elements of a story. Based on these two elements there are currently two research directions: plot-based and character-based interactive storytelling. As interactive storytelling is a mixture of conventional storytelling and user interactions, and due to the tradeoff between story narrative and user interactions, striking a balance between the two is a big challenge. Consequently, there is a need to develop an integrated framework to achieve the balance between conveying a story's moral and enhancing the modeling of character behaviors. As a solution to such a challenge, Bcreative system was developed. It is a unified prototype of both plot-based and character-based interactive storytelling. It gives the opportunity for the user to get all the ideas out of his mind and be creative, hence the name Bcreative. This occurs in a short time, in a simple way with great efficiency, and with no budget by manipulating the embedded smart user-friendly graphical user interface. The idea of Bcreative is derived from the study of Petri nets, cinematography in interactive storytelling, and filmmaking theory.

\subsection{Prior Research}

Recent developments in interactive storytelling have resulted in many different approaches which differ on various dimensions and sometimes overlap, such as: immersive storytelling [12]; emergent storytelling [1]; interactive story authoring/generation [10]; plot-based [7,13,17] and character-based interactive storytelling [2,3]. Pérez y Pérez, R. \& Sharples, M. [16] present an updated review of different interactive 
storytelling systems with comparisons. More recently, a hybrid system of plot-based and character-based interactive storytelling is discussed in [19]. One of the characteristics of the previous approaches is in controlling a balance between interaction and narration. They showed a lot of interesting results. However, they didn't consider a user's level of understanding, even though people have different abilities to understand a story according to their knowledge, experience, age, gender, etc. Moreover, previous work has identified relevant dimensions and key problems for the implementation of interactive storytelling, among which are: the status of the user, the level of explicit narrative representation and narrative control, the modes of user intervention, the duality between character and plot, the tension between interactivity and storytelling, etc. This paper attempts to address these problems, in particular, the relations between character and plot by modeling the story plot as well as the character behavior in an integrated framework through a smart-user friendly graphical user interface (GUI) platform called Bcreative.

\section{Proposed System Development Process}

Developing an interactive storytelling system to achieve the autonomy of story generation and story performing is an exhaustive and complicated process requiring careful design and layout of the story content and its visual presentation. This section discusses the development process of our proposed system from the earlier design phase called the process model up to the final product system.

\subsection{Methodology and System Workflow}

The methodology in our system is based on a basic ontology which holds the various concepts that are relevant to story plot generation as well as character behavior. The system architecture is a composition of two engines: story engine and character engine through a smart user-friendly graphical user interface. The story engine models the story plot based on the PN reachability analysis [5], while the character engine enhances the modeling of character behavior based on cinematography in interactive storytelling, and the story world elements (e.g. actors, camera, lighting, etc.). The system workflow is shown in Fig. 1, where the content inside the oval shape represents an output from the previous task or state.

Based on the circumstances that a story scene occurs (story context), Fig. 1 shows that the TV content/story is produced from a set of various meaningful storylines or plots, each of which is composed of scenes, which are modeled based on PN reachability, and will be explained in detail in subsection 4.3. In our approach, we used Petri nets (PN) as a state-based model for story plot, where each scene in a plot is regarded as a goal to be reached. A complex scene is represented as a composite goal, and a simple scene is represented as an atomic goal. The director (Bcreative user) acts as the storyteller, who dynamically selects and determines the storyline from the whole plot based on the user interaction or context variables. A scene of the storyline is composed of beats. A beat is a dramatic action or the smallest story unit that occurs in a scene to achieve a narrative goal. A beat in our system consists of an action/reaction pair between actors and will be generated in real-time using a character 


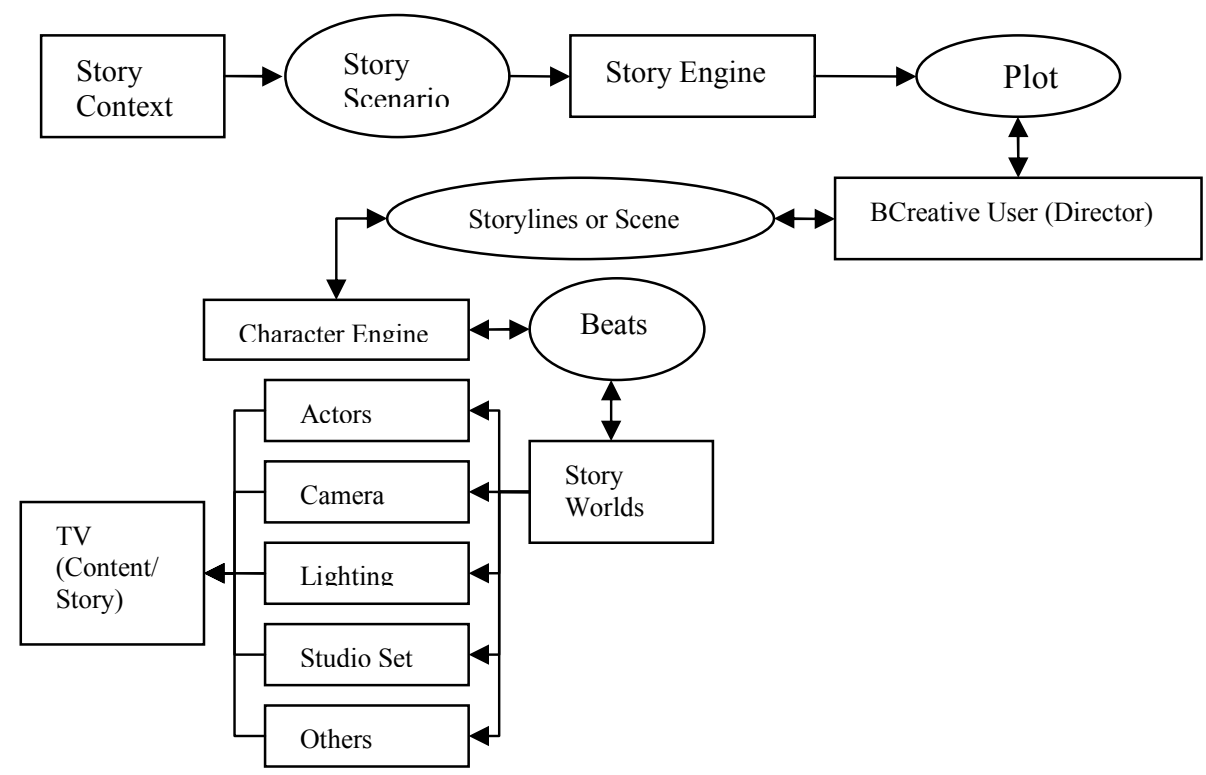

Fig. 1. System Workflow

engine and executed through artistic works called story worlds via its dramatic components (e.g. actors, camera, etc.). For a complex scene in the presentation path, the goal can be decomposed into more specific sub-goals. Depending on the user interactions and context, different consequent goals may be reached after a certain goal, i.e. different scenes are achieved in different situations.

\subsection{Architectural Design and Graphical User Interface (GUI)}

Design involves decomposing the system into modules. The result is a design specification document which contains a description of the software architecture: what each

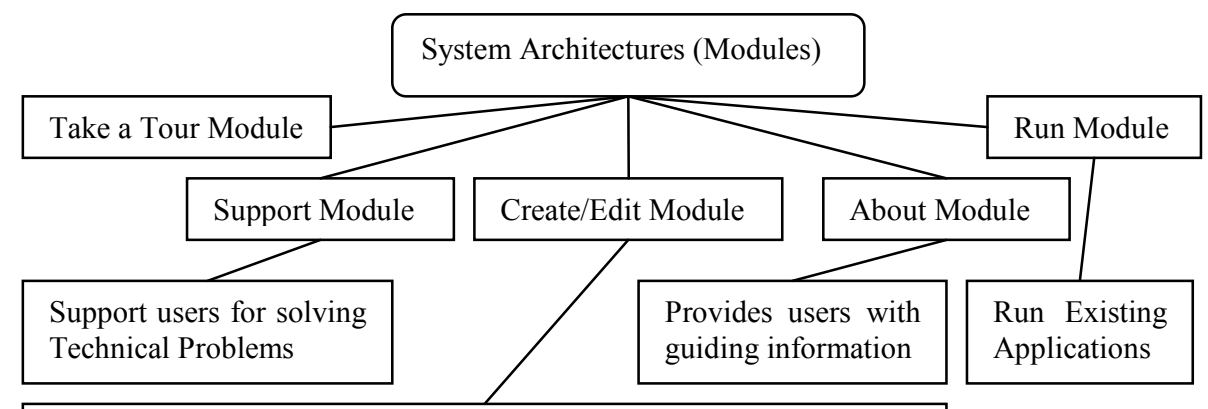

Edit an existing application and/or creates a new plot by setting the necessary story worlds based on some particular story context

Fig. 2. Architectural Design 
module is intended to do and the relationships among modules. Fig. 2 shows some of the basic architectural design of our proposed storytelling framework. Fig. 3 shows these different modules via the main graphical user interface (GUI) screen window once we run the Bcreative system.

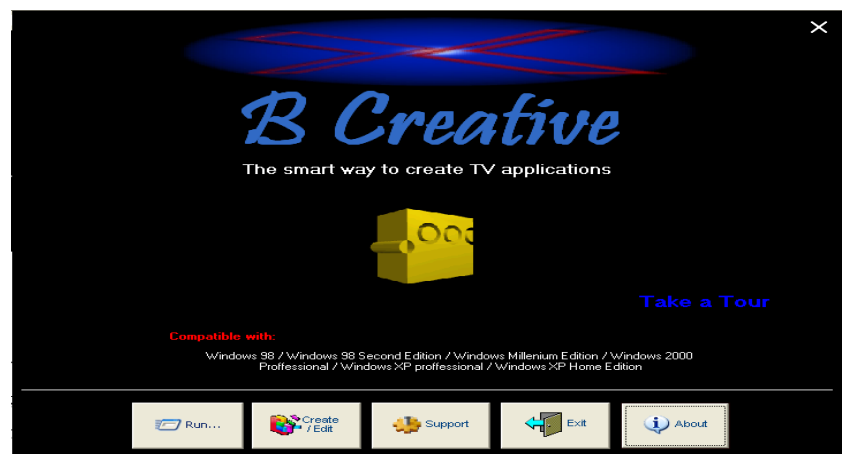

Fig. 3. Bcreative Main (GUI) Screenshot

\subsection{Story Engine}

Reachability is the fundamental basis for studying the dynamic properties of any system [5]. The firing of an enabled transition will change the token distribution in a Petri nets according to the transition firing rules described in subsection 2.3. The idea behind using Petri nets (PN) to model the story plot as well as character behavior is based on three factors: (i) the story is generated from a set of smaller sub-stories or plots, each of which is composed of scenes, and scenes are composed of beats, (ii) the degree of freedom, which the PN possesses (e.g. marking of the net, the sequence of firing, etc.) as well as PN properties such as, sequential execution, conflict, concurrency, confusion, inhibitor arcs to model priorities, etc., and (iii) the concept of PN reachability which can be simply stated as "Can we reach one particular state from another?". Based on these factors, our idea is derived for using PN as a state-based model for story representation/plot. Each scene in a plot is represented as a graph of desired states to be reached. The final goal states for a scene mark the end of the scene, just as the initial state describes how the scene should begin. A state is marked with a transition from one state to another, which provides a relative ordering of how states should flow temporally. Transitions are "source-action-target" triplets where the execution of an action triggers a change of state. For the state of a Petri nets only the set of places $\mathrm{P}$ is relevant, because the network structure of a Petri nets does not change and only the distribution of tokens over places changes. A state, also referred to as marking, corresponds to a mapping from places to natural numbers. Marking is based on the number of beats the scene constitutes, and plots are constructed based on the firing the beat, where a scene of the story is regarded as a goal. Any state s can be presented as $s \in P \rightarrow\{0,1,2, \ldots\}$, i.e., a state can be considered as a multi-set, function, or vector. In the context of state spaces, we use places as attributes. In any state the value of each place attribute is known: $s(p)$ is the value of attribute $p \in P$ in 
state $\mathrm{s}$. Since, firing a transition will change the state marking $M$ to a new mark$\operatorname{ing} M^{\prime}$. The state space of a Petri nets with $\mathrm{n}$ places is the set of all markings, that is $N^{n}$. Now the question that arises is how to decide which scene to attempt to make happen next? For this, we should examine the list of unused scene and choose the one that has a satisfied precondition and whose value change best matches the shape of the global plot arc. We formulated the state change caused by a transition firing using a partial function $\delta$ which we call the next-state function, where,

$$
\delta: N^{n} \times T \rightarrow N^{n}
$$

The function $\delta$ when applied to a marking $\mathrm{M}$ and a transition $t_{j}$ yields the new marking $M^{\prime}$ which results from firing the enabled transition $t_{j}$ in the marking $\mathrm{M}$, that is,

$$
\delta\left(M, t_{j}\right)=\left\{\begin{array}{cccc}
\text { undefined } & \text { if } & t_{j} & \\
& \text { is } & \text { not } & \text { enabled } \\
M^{\prime} & \text { if } & t_{j} & \\
& \text { is } & & \text { enabled }
\end{array}\right.
$$

$M^{\prime}$ is the marking which results from removing tokens from the inputs of $t_{j}$ and adding tokens to the output of $t_{j}$. The function $\delta$ given by equation (2) incorporates a notion of distributed state, and a rule for state change of a Petri net via a sequence of markings $\left(M_{0}, M_{1}, M_{2}, \ldots\right)$ and a sequence of transitions $\left(t_{j(0)}, t_{j(1)}, t_{j(2)}, \ldots\right)$ which were fired. The relationship between these two sequences as well as the properties of Petri nets [18] such as, sequential execution, conflict, concurrency, confusion, duality, conflict, zero-testing through the introduction of inhibitor arcs to model priorities etc., form the main idea for story engine to model the story plot. An inhibitor arc connects a place to a transition and is represented by a dashed line terminating with a small circle instead of an arrowhead at the transition. The introduction of inhibitor arc adds the ability to test "zero" (i.e. absence of tokens in a place). The notion of conflict, on the other hand, is often described as the main mechanism of drama, where it occurs when a possible action for a character is not compatible with his/her value, e.g. a solitary boy must approach a girl, an aristocratic lady can't reveal her love for a poor servant, etc. Fig. 4(a-e) demonstrates some of the (PN) properties such as sequential execution, conflict, concurrency, confusion, etc. which are represented using a set of simple constructs as follows:

A. Sequential execution: (Fig. 4(a)), transition $t_{2}$ can fire only after the firing of $t_{1}$. This imposes the precedence of constraints " $t_{2}$ after $t_{1}$ ".

B. Conflict: Transitions $t_{1}, t_{2}$ and $t_{3}$ are in conflict as shown in Figure 4(b). All are enabled but the firing of any leads to the disabling of the other transitions. 
C. Concurrency: (Fig. 4(c)), the transitions $t_{1}, t_{2}$, and $t_{3}$ are concurrent. A necessary condition for transitions to be concurrent is the existence of a forking transition that deposits a token in two or more output places.

D. Priorities: (Fig. 4(d)), the classical Petri nets have no mechanism to represent priorities. Inhibitor nets defined special arcs called inhibitor arcs to model priorities.

E. Confusion: A situation where concurrency and conflicts co-exist (Fig. 4(e)). Both $t_{1}$ and $t_{3}$ are concurrent, while $t_{1}$ and $t_{2}$, are in conflict. Also, $t_{2}$ and $t_{3}$ are in conflict.

Equation (2) can be written in a compact recursive manner as follows:

$$
\delta\left(M_{k}, t_{j(k)}\right)=M_{k+1} \text { for } \mathrm{k}=0,1,2,
$$

Equation (3) forms an iterative process of marking which exhibits a certain analogy of the recursive process for story plot modeling. Fig. 5 shows a sample story scenario example for equation (3), which contains scenes from $P_{1}$ (start state) to $P_{4}$ (final or goal state) with initial marking $M_{0}=(1,0,1,0,2)$.

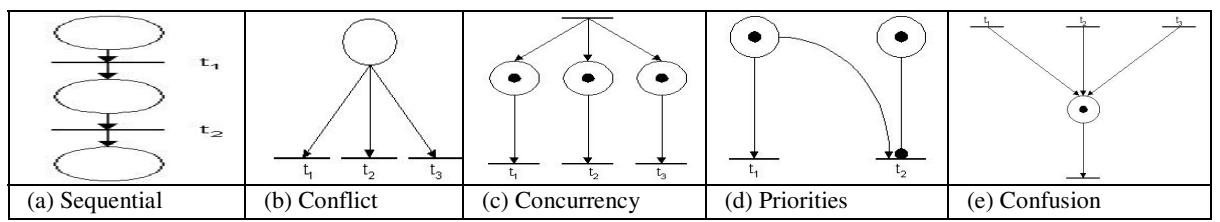

Fig. 4. Some PN Properties illustrations

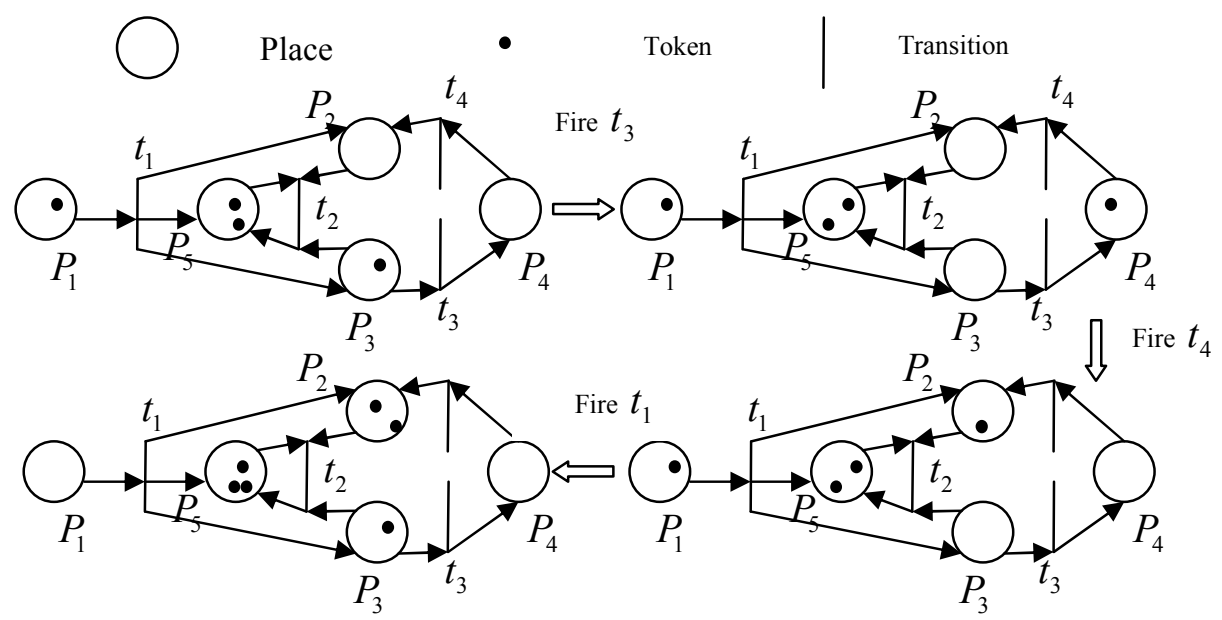

Fig. 5. Scene Scenarios Example with Marking Reachability 
In Fig. 5, tokens are regarded as beats where marking is based on. For instance, with the initial marking $M_{0}=(1,0,1,0,2)$, two transitions $t_{1}$ and $t_{3}$ are enabled, choosing $t_{3}$ producing the marking $\delta\left(M_{0}, t_{3}\right)=(1,0,0,1,2)=M_{1}$. In this marking, transitions $t_{1}$ and $t_{4}$ are enabled, firing $t_{4}$ changes the marking to $\delta\left(M_{1}, t_{4}\right)=(1,1,0,0,2)=M_{2}$. In $M_{2}, t_{1}$ is enabled and firing it leads to $\delta\left(M_{2}, t_{1}\right)=(0,2,1,0,3)=M_{3}$.

\subsection{Character Engine}

Based on the user interactions or context, the director (Bcreative user) dynamically selects and determines the scenes from the whole plot obtained previously from story engine, and then assigns the scene contents folded by its dramatic components (e.g. camera, lighting, etc.) to different actors involved in the scene via the character engine and based on filmmaking theory. Story plot and character interactions are two important elements of interactive storytelling. However, we believe that interactive stories also need to include investigation dealing with the visual presentation of the story and the way it is shown and told. Theatre directors, filmmakers, and animators have emphasized the importance of visual design. Film and theatre, as well as animation artists, spend many hours, days, or even months, creating a visual design for a production. A visual design is considered the heart of a performance; it deepens and enriches the dramatic experience through artistic works called story worlds via its dramatic components which have direct impact on communicating the narrative, evoking emotions and moods, and engaging viewers. These components have many psychological and aesthetic effects that influence viewers' perception of a scene. We considered these important factors in developing our system and modeled the process of the character engine according to program-production processes used in the real world. Character engine deals with the visual presentation of the story and the way it is shown and told, in the context of applying a formal cinematic language to the visual components, in order to give a better shape to the story, and to the whole dramatic experience [4,9]. Cinematic language, translated from a movie to a virtual environment, should be a valid option, with different proved formalisms that can be translated to a storytelling system. Our system embedded a modified TVML [6] as a cinematic language for an action/reaction pair between actors, executed through story worlds via its dramatic components in order to provide better dramatic experiences and supporting the narrative relevance of the stories being narrated. The character engine structure follows standard formats for content production which is classified into two categories: "information data" and "presentation data". Information data refers to the information that is to be conveyed to viewers via the program. Presentation data describes the way in which that information should be shown to viewers as a program. Presentation data is the core of any content production which needs some resources or production modules obtained from the information data. Character engine contains two modes: scene mode and editing mode. Both of them are supported by a userfriendly graphical user interface (GUI) screens. Screenshots for some of these screens are shown in Fig. 6. Each screen supports level of details (LOD) rendering techniques. The user starts in scene mode to execute his/her scene constructions (tasks) 
required for creating the desired shots based on the information data and/or the story plot given. The user then moves to editing mode to edit, run, manipulate, play movies or preview the shots which have been previously constructed. Character engine architecture is shown in Fig. 7.
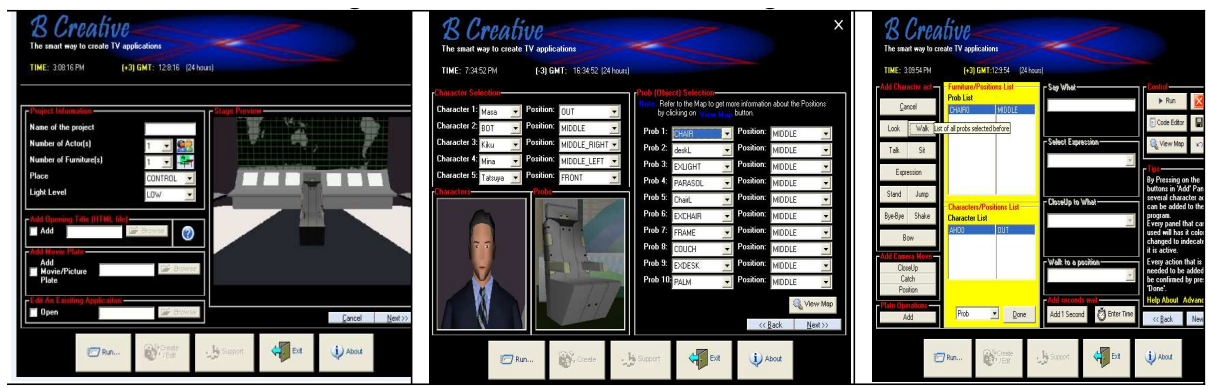

Fig. 6. Screen shoots of character engine GUI supporting screens

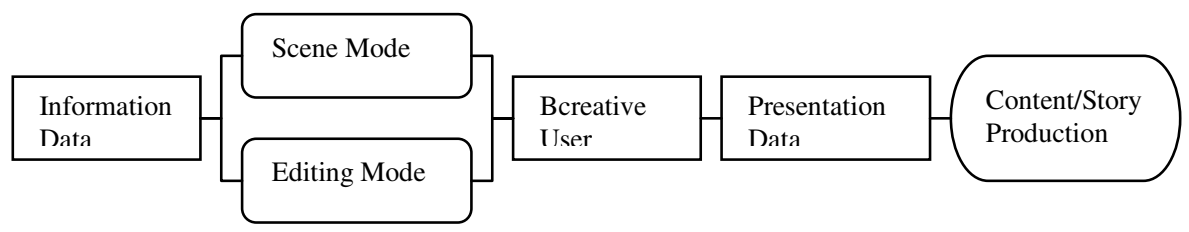

Fig. 7. Character Engine Structure

\section{System Configuration}

Bcreative environment is a general purpose TV content/story production application system. It is compatible with different Windows operating systems. The hardware environment requires computers with multimedia support. The software environment is based on Microsoft Visual studio tools, Microsoft DirectX 9 drivers, TTS (Text to Speech) engine, and the multimedia tool called TVML [6]. TVML is a scripting language used for story world and embedded in our system. Unfortunately, TVML involves a limited number of characters; therefore, the system may allocate one character to multiple actors, which confuses the viewers. In our implementation, we solved this problem by increasing the number of characters as well as probes usable in TVML. The Bcreative user doesn't need to understand the TVML (more than 90 commands) because our system supports the user with a smart user-friendly screens.

\section{Evaluation and Performance Results}

\subsection{User Test}

The functionality and the performance of the proposed system have been evaluated according to several criteria such as: (i) the creativity process including psychological 
and historical creativity, story-predictability including structure and content elements, (ii) story-development processes and knowledge data-bases stored by the system, including rhetoric-knowledge, story-world knowledge and common-sense knowledge, (iii) interestingness which is a combination of content, originality, suspense and coherence (the user's ability to comprehend the relationships between the events in the story, both within the story world and storytelling), and (iv) use of the natural language structure and meaning. These criteria are the elements of literary criticism that cannot be captured by objective measures. The system was shown to a few visual artists and designers who are practically test the system and reported the following results obtained for the story (in a scale from 1 to 5 , where 5 indicates best): narrative flow: 3.9; clever plot: 4.1; coherency: 4.5; use of language: 4.4; content: 3.9; suspense: 4.2 ; creativity: 4.7 . They showed great interest in the system, especially in its use as a rapid prototyping tool for writers. They reported that the system has shown great utility as an authoring tool for content production and can be objectively used as a teaching aid for many purposes like education, cinematography, communication, etc. Comparisons with existing systems showed that the Bcreative system process is similar to the Minstrel model [16] in knowledge structures where they are entered by a graphical interface that allows dropping frames from a menu and interconnecting them with relationships. Similarly to the Mexica model [15]; its story-predictability can be classified as low. In addition, it ensures novelty most like both Minstrel and Mexica models. Table 1 compares the Bcreative system with some other existing systems like Façade [11], I-storytelling [20], and hybrid system [19].

Table 1. System Comparisons with other system models

\begin{tabular}{|c|c|c|c|c|}
\hline Factor & Facade & $\begin{array}{c}\text { I- } \\
\text { Storytelling }\end{array}$ & Hybrid & Bcreative \\
\hline Description & $\begin{array}{l}\text { Credible } \\
\text { characters } \\
\text { controlled by a } \\
\text { dramatic } \\
\text { manager. }\end{array}$ & $\begin{array}{l}\text { Virtual actors } \\
\text { with dramatic } \\
\text { goals }\end{array}$ & $\begin{array}{l}\text { Multi-agent storytelling } \\
\text { system }\end{array}$ & $\begin{array}{l}\text { An authoring environment for } \\
\text { virtual storytelling }\end{array}$ \\
\hline Methodology & $\begin{array}{l}\text { ABL } \\
\text { program- } \\
\text { ming, beat, } \\
\text { dramatic } \\
\text { arch. }\end{array}$ & $\begin{array}{l}\text { Hierarchical } \\
\text { Task } \\
\text { Network } \\
\text { (HTN) } \\
\text { planning }\end{array}$ & $\begin{array}{l}\text { Fuzzy Cognitive Goal Net } \\
(\text { FCGN) }\end{array}$ & $\begin{array}{l}\text { Petri nets, filmmaking theory } \\
\text { and cinematography in } \\
\text { interactive storytelling }\end{array}$ \\
\hline Technology & $\begin{array}{l}\text { Java \& JESS } \\
\text { reasoning. }\end{array}$ & $\begin{array}{l}\text { Unreal and } \\
\mathrm{C}++ \\
\text { planning }\end{array}$ & $\begin{array}{l}\text { Active Worlds 3-D virtual } \\
\text { environment powered by } \\
\text { Renderware }\end{array}$ & $\begin{array}{l}\text { Microsoft visual studio tools, } \\
\text { TVML, and other multimedia } \\
\text { tools }\end{array}$ \\
\hline Application & $\begin{array}{l}\text { An interactive } \\
\text { drama. }\end{array}$ & $\begin{array}{l}\text { Interactive } \\
\text { television }\end{array}$ & Interactive storytelling & $\begin{array}{lll}\begin{array}{l}\text { Interactive } \\
\text { production }\end{array} & \text { TV } & \text { content/story } \\
\end{array}$ \\
\hline Scalability & Intermediate & Intermediate & Intermediate & High \\
\hline Replayability & High & High & Intermediate & High \\
\hline Creativity & High & Intermediate & Intermediate & High \\
\hline $\begin{array}{l}\text { Natural lang. } \\
\text { production }\end{array}$ & High & Intermediate & Low & High \\
\hline $\begin{array}{l}\text { Graphical user } \\
\text { Interface (GUI) }\end{array}$ & Intermediate & Intermediate & Low & High \\
\hline Interestingness & High & High & High & High \\
\hline
\end{tabular}




\subsection{Case Study}

One of several real case studies which have been done for testing our system is based on a story scenario of horse riding (Endurance \& Jumping). Horse riding is an interesting and famous sport worldwide [8]. We tested our system for producing TV content/story of horse riding as a teaching aid for beginners. Fig. 8 shows the screen shots for such content production. The corresponding Petri nets used to model the story scenario is shown in Fig. 9.
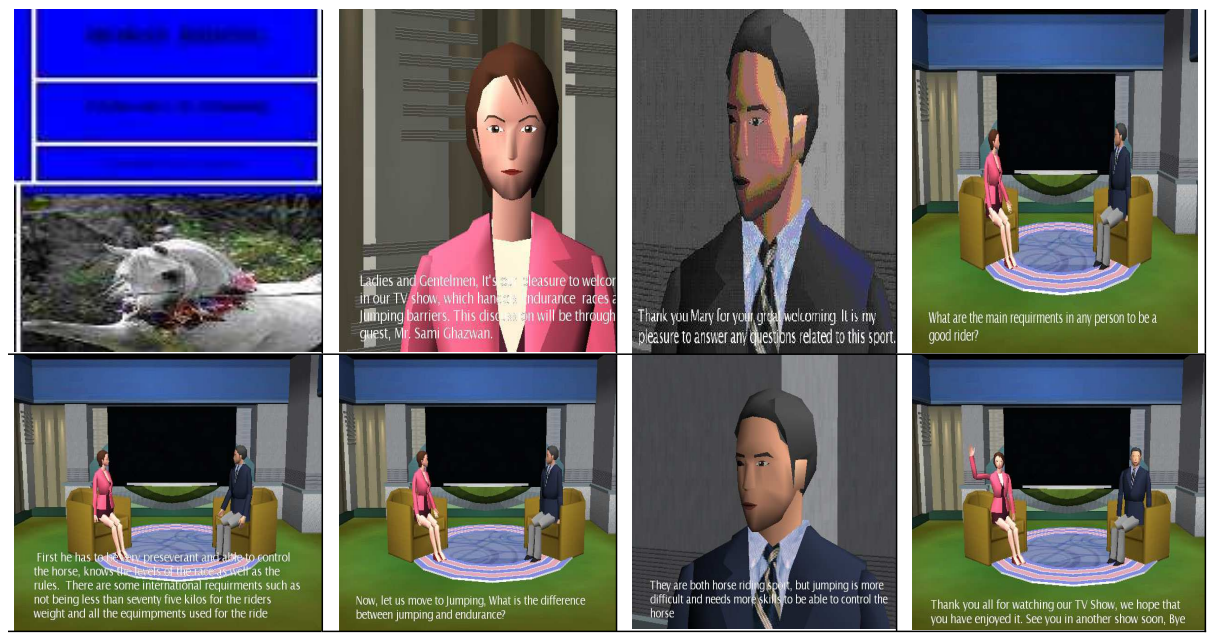

Fig. 8. Horse riding content/story production example created by our System

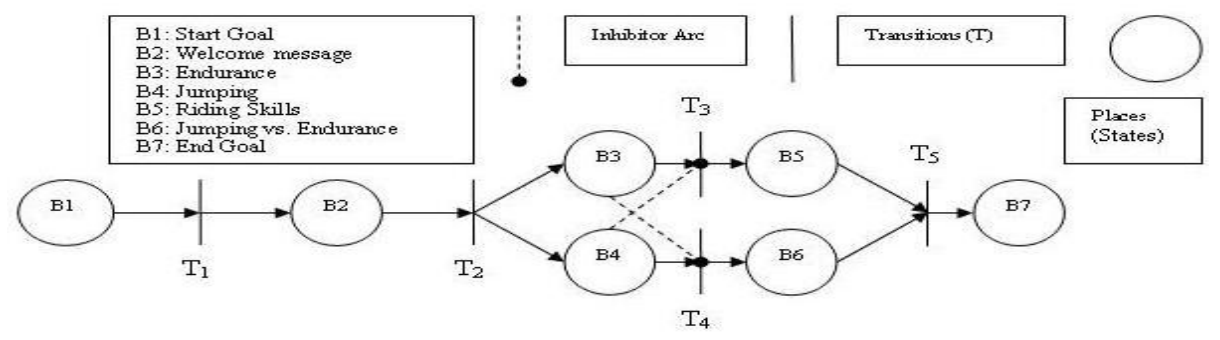

Fig. 9. Corresponding PN for scene scenario of figure 7 with beats B

\section{Conclusion and Future Work}

TV content/story production is a major bottleneck in the entertainment industry. In this paper, we have proposed an authoring environment for virtual storytelling which attempts to reconcile the tension between interactivity and storytelling as well as narrative progression and user interaction. The developed system is composed of two engines: a story engine and a character engine through a smart user-friendly graphical user interface. The story engine models the story plot based on the PN reachability 
analysis, while the character engine enhances the modeling of character behavior based on filmmaking theory. The proposed system can be objectively used as a teaching aid for many purposes like education, cinematography, communication, etc. The merits and the original contribution of this paper are:

1) Presenting a new idea for interactive storytelling technology using a new kind of analysis method for the marking of the PN and its reachability analysis. We show that PN as a graphical and modeling tool can be used as a visual-communication aid in films to model the computer graphics scenes (story plot) and characters.

2) Reporting that most computer-based application techniques developed outside the realm of Petri nets can be applied to Petri nets as well.

3) Developing an integrated framework of both plot-based and character-based interactive storytelling to achieve the balance between conveying a story and enhancing the modeling of character behaviors as well as reconcile the character-based approach with the problem of narrative control.

4) Enhancing scalability issues, which are satisfied by distinguishing the story beats and their visual presentation, where this abstraction has enhanced scalability and promoted reuse by enabling the reuse of the same visual presentation with different story beats.

5) Supporting replayability (entertainment value), the creativity process and others.

Creativity is a fascinating area where there are too many things waiting to be discovered. Hopefully, this paper will encourage further work on computer models of creativity. Despite the great efficiency of the obtained results, currently we are working on extending our system to be applicable in multi-language with speed animation enhancement.

\section{References}

[1] Aylett, R.: Narrative in Virtual Environments: towards emergent narrative. In: AAAI 1999 Fall Symposium on Narrative Intelligence (1999)

[2] Cavazza, M., Charles, F., Mead, S.J.: Interacting with Virtual Characters in Interactive Storytelling. In: ACM Joint Conference on Autonomous Agents and Multi-Agent Systems, Bologna, Italy, pp. 318-325 (2002)

[3] Cavazza, M., Charles, F., Mead, S.J.: Character-based Interactive Storytelling. IEEE Intelligent Systems, special issue on AI in Interactive Entertainment, pp. 17-24 (2002)

[4] Courty, N., Lamarche, F., Donikian, S., Marchand, E.: A cinematography system for virtual storytelling. In: Balet, O., Subsol, G., Torguet, P. (eds.) ICVS 2003. LNCS, vol. 2897, pp. 30-34. Springer, Heidelberg (2003)

[5] Huang, J., Tadao, M.: Classification of Petri net transitions and their application to firing sequence and reachability problems. In: IEEE Conference on Systems, Man, and Cybernetics, vol. 1, pp. 263-268 (1997)

[6] Hayashi, M., Douke, M., Ariyasu, K., Hamaguchi, N.: TV program Making language Benefits of object-based contents-creation method. In: IBC 2001 Conf., pp. 23-31 (2001)

[7] Hussein, H.K.: A new framework for Plot-based interactive storytelling generation. In: 5th IEEE International Conference Computer Graphics, Imaging and Visualization, Malaysia, Pennang, August 25-28 (accepted) (to appear, 2008) 
[8] Kijima, R., Kouno, M., Hashimoto, K., Jiang, Y., Aoki, T., Ojika, T.: Karakuri Horse Riding Therapy. In: Proc. of the Int. Conf. on Rehabilitation Robotics, pp. 278-281 (2003)

[9] El-Nasr, M.S.: Interactive narrative architecture based on filmmaking theory. International Journal on Intelligent Games and Simulation 3(1) (2004)

[10] Magerko, B., Laird, J.: Building interactive drama architecture. In: First Int. Conf. on Technologies for Interactive Digital Storytelling and Entertainment, pp. 226-237 (2003)

[11] Mateas, M., Stern, A.: Façade: An experiment in building a fully-realized Interactive Drama. In: Game Developers Conference, Game Design track (2003)

[12] Nakatsu, R., Tosa, N.: Interactive Movies. Handbook of Internet and Multimedia. IEEE, Los Alamitos (2000)

[13] Pablo, G., Díaz-Agudo, B., Federico, P., Raquel, H.: Story plot generation based on CBR. Knowledge-Based Systems Journal 18, 235-242 (2005)

[14] Peterson, J.L.: Petri nets. ACM Computing Surveys 9(3), 223-252 (1977)

[15] Perez y Perez, R., Sharples, M.: MEXICA: A computer model of a cognitive account of creative writing. Journal of Experimental and Theoretical AI (2001)

[16] Perez y Perez, R., Sharples, M.: Three computer-based models of storytelling: BRUTUS, MINSTREL and MEXICA. Knowledge Based Systems Journal 17(1) (2004)

[17] Sgouros, N., Papakonstantinou, G., Tsanakas, P.: A Framework for Plot Control in interactive story systems. In: Proc. Am. Assoc. Artificial Intelligence (AAAI 1996), pp. 162 167. AAAI Press, Menlo Park (1996)

[18] Tado, M.: Petri nets: properties, analysis and applications. Proc. IEEE 77 (1990)

[19] Cai, Y., Miao, C., Tan, A.-H., Shen, Z.: A Hybrid of Plot-Based and Character-Based Interactive Storytelling. In: Hui, K.-c., Pan, Z., Chung, R.C.-k., Wang, C.C.L., Jin, X., Göbel, S., Li, E.C.-L. (eds.) EDUTAINMENT 2007. LNCS, vol. 4469, pp. 260-273. Springer, Heidelberg (2007)

[20] I-storytelling, http: / /www-scm.tees.ac.uk/users/f . charles/ 